\title{
TOWARD A PRECISE SMOOTHNESS HYPOTHESIS IN SARD'S THEOREM
}

\author{
S. M. BATES
}

(Communicated by Charles Pugh)

\begin{abstract}
The familiar $\mathbf{C}^{k}$ smoothness hypothesis in the Morse-Sard Theorem can be weakened to $\mathbf{C}^{k-1,1}$.
\end{abstract}

\section{INTRODUCTION}

Let $f: \mathbf{R}^{n} \rightarrow \mathbf{R}^{m}$ be a differentiable mapping. A question of fundamental importance in differential topology and dynamical systems concerns the measure of the critical value set of $f$, i.e., the image under $f$ of those points $x \in \mathbf{R}^{n}$ such that $D f(x)$ is not surjective. The basic theorem regarding this problem is due to Morse and Sard (see $[9,10])$ :

The Morse-Sard Theorem. Let $f: \mathbf{R}^{n} \rightarrow \mathbf{R}^{m}$ be a $\mathbf{C}^{k}$ map. If $k \geq$ $\max \{n-m+1,1\}$, then the set of critical values of $f$ has Lebesgue m-measure zero.

The necessity of the above differentiability requirement was established by Whitney [11], who constructed a $\mathbf{C}^{1}$ map $f: \mathbf{R}^{2} \rightarrow \mathbf{R}$ not constant on a connected set of critical points (see also $[1,2,4,5]$ ). Since the appearance of Whitney's example, investigations of the geometry of critical sets and critical values have led to various more precise formulations of the Morse-Sard Theorem (e.g., $[3,7,8,12])$.

A map $f \in \mathbf{C}^{p}\left(\mathbf{R}^{n}, \mathbf{R}^{m}\right)$ is said to belong to $\mathbf{C}^{p, 1}$ if $D^{p} f$ is locally Lipschitz on $\mathbf{R}^{n}$. One object of this paper is to prove

Theorem 1. Let $n, m$ be positive integers with $n>m$ and $k=n-m+1$. If $f \in \mathbf{C}^{k-1,1}\left(\mathbf{R}^{n}, \mathbf{R}^{m}\right)$ then the set of critical values of $f$ has $m$-measure zero.

Thus the differentiability requirement in the classical Morse-Sard Theorem can be relaxed from $\mathbf{C}^{k}$ to $\mathbf{C}^{k-1,1}$.

For $\alpha \in(0,1)$, recall that $f \in \mathbf{C}^{p}\left(\mathbf{R}^{n}, \mathbf{R}^{m}\right)$ is said to be of (Hölder) smoothness class $\mathbf{C}^{p, \alpha}=\mathbf{C}^{p+\alpha}$ if for every compact $B \subset \mathbf{R}^{n}$, there exists a real constant

Received by the editors May 24, 1991.

1991 Mathematics Subject Classification. Primary 58C25, 58C27; Secondary 26C35, 57R70.

Supported by an NSF graduate fellowship in Mathematics. 
$M$ such that

$$
\left\|D^{p} f(x)-D^{p} f(y)\right\| \leq M|x-y|^{\alpha}
$$

for all $x, y \in B$. A subset $E \subset \mathbf{R}^{n}$ is called a set of rank $r$ for $f$ provided rank $D f(x) \leq r$ for all $x \in E$. The central theorem of this paper is

Theorem 2. Let $n, m$, and $r$ be nonnegative integers satisfying $n>m>r$, and define $s=(n-r) /(m-r)$. If $E$ is a set of rank $r$ for $f: \mathbf{R}^{n} \rightarrow \mathbf{R}^{m}$ and either

(a) $s \in \mathbf{Z}^{+}$and $f \in \mathbf{C}^{s-1,1}$ or

(b) $f \in \mathbf{C}^{s}$,

then $f(E)$ has m-measure zero.

Note that by substituting $r=m-1$ into the statement of Theorem 2, one obtains Theorem 1.

We remark that Norton $[7,8]$ has proven Theorem 2 under more stringent smoothness requirements or, alternatively, under the assumption that $E$ has $n$ measure zero. Theorem 2(a) is stronger than Federer's Theorem 3.4.3 [3], which implies the same conclusion for integer $s$ provided $f \in \mathbf{C}^{s}$. Theorem 2(b) improves the estimates given by Yomdin's Theorem 5.3 [12], which concludes that the entropy dimension (and hence Hausdorff dimension) of $f(E)$ is at most $m$.

The degree to which Theorem 2 is itself sharp is illustrated by the following fact, proven by the author in [2].

Theorem 3. For $n, m, r, s$ as in Theorem 2, there exists a map $f: \mathbf{R}^{n} \rightarrow \mathbf{R}^{m}$ contained in $\mathbf{C}^{t}$ for all real $t<s$ and a subset $E$ of rank $r$ for $f$ such that $f(E)$ contains an open set.

Thus any further improvement of Theorem 2 and in particular of the MorseSard Theorem must distinguish between $\mathbf{C}^{p, 1}$ and smoothness classes contained in $\mathbf{C}^{t}$ for all $t<p+1$.

Finally, we note that an application of Theorem 2 to singular mappings appears in [2].

We denote by $l_{m}$ the Lebesgue outer measure on $\mathbf{R}^{m}$. A subset $E \subset \mathbf{R}^{m}$ is called $m$-null, resp. $m$-finite, provided $l_{m}(E)=0$, resp. $l_{m}(E)<\infty$.

\section{Proof of Theorem 2}

A crucial observation is that the proof of Theorem 2 reduces to the case $r=0$; accordingly, our goal in the next section is to prove

Lemma 1. Let $n, m$ be positive integers with $n>m$, and define $v=n / m$. If $E \subset \mathbf{R}^{n}$ is a set of rank 0 for $f: \mathbf{R}^{n} \rightarrow \mathbf{R}^{m}$ and either

(a) $v \in \mathbf{Z}^{+}$and $f \in \mathbf{C}^{v-1,1}$ or

(b) $f \in \mathbf{C}^{v}$,

then $f(E)$ is m-null.

Assuming Lemma 1 for the time being, we now derive Theorem 2(a) following Sard and Norton:

For $i=0,1, \ldots, r, r<m$, define $R_{i}=\{x \in E: \operatorname{rank} D f(x)=i\}$; we show $l_{m} f\left(R_{i}\right)=0$ for each $i$.

(1) $l_{m} f\left(R_{0}\right)=0$ by Lemma $1(\mathbf{a})$, since $v \leq s$ and $f$ is $\mathbf{C}^{s-1,1}$. 
(2) Fix $i \in\{1, \ldots, r\}$ and $p \in R_{i}$. It will suffice to find a neighborhood $V$ of $p$ such that $f\left(V \cap R_{i}\right)$ is $m$-null. By the $\mathbf{C}^{s-1,1}$ Inverse Function Theorem (see $[7,8]$ ), there are coordinates in some neighborhood $V$ of $p$ such that

$$
f\left(x_{1}, \ldots, x_{n}\right)=\left(x_{1}, \ldots, x_{i}, g\left(x_{1}, \ldots, x_{n}\right)\right),
$$

where $g \in \mathbf{C}^{s-1,1}\left(\mathbf{R}^{n}, \mathbf{R}^{m-i}\right)$. In these coordinates,

$$
D f(y)=\left(\begin{array}{cc}
\operatorname{Id}_{i} & 0 \\
* & D\left(g \| x_{1}, \ldots, x_{i}\right)
\end{array}\right),
$$

where $\operatorname{Id}_{i}$ is the identity matrix on $\mathbf{R}^{i}, y=\left(x_{1}, \ldots, x_{n}\right)$, and $g \| x_{1}, \ldots, x_{i}$ : $\mathbf{R}^{n-i} \rightarrow \mathbf{R}^{m-i}$ is the mapping defined by $\left(x_{i+1}, \ldots, x_{n}\right) \mapsto g\left(x_{1}, \ldots, x_{n}\right)$. Note that $D\left(g \| x_{1}, \ldots, x_{i}\right)$ has rank 0 for all $y \in R_{i}$.

For $S \subset \mathbf{R}^{n}$ and $\left(x_{1}, \ldots, x_{i}\right) \in \mathbf{R}^{i}$, we denote by $S\left[x_{1}, \ldots, x_{i}\right]$ the set of points $\left(x_{i+1}, \ldots, x_{n}\right) \in \mathbf{R}^{n-i}$ such that $\left(x_{1}, \ldots, x_{n}\right) \in S$ (this is the "slice" of $S$ through $\left.\left(x_{1}, \ldots, x_{i}\right)\right)$. Then $g \| x_{1}, \ldots, x_{i}$ maps the rank 0 set $\left(V \cap R_{i}\right)\left[x_{1}, \ldots, x_{i}\right]$ onto $\left(f\left(V \cap R_{i}\right)\right)\left[x_{1}, \ldots, x_{i}\right]$.

Because $(n-i) /(m-i) \leq(n-r) /(m-r)=s$ and $g \| x_{1}, \ldots, x_{i}$ is $\mathbf{C}^{s-1,1}$, it follows from Lemma 1 (a) that every slice of $f\left(V \cap R_{i}\right)$ is $(m-i)$-null in $\mathbf{R}^{m-i}$. Applying Fubini's Theorem to $\mathbf{R}^{i} \times \mathbf{R}^{m-i}$, we conclude that $f\left(V \cap R_{i}\right)$ is $m$-null, and Theorem 2(a) follows.

To prove Theorem 2 (b), one proceeds analogously, replacing $\mathbf{C}^{s-1,1}$ with $\mathbf{C}^{s}$ and Lemma 1(a) with Lemma 1(b) throughout the above argument.

\section{Proof of Lemma 1}

In order to prove Lemma 1, we require the following result due to Norton.

Lemma 2 (Norton). Let $n, m$ be positive integers with $n>m$, and define $v=n / m$. If $E$ is an n-null set of rank 0 for $f: \mathbf{R}^{n} \rightarrow \mathbf{R}^{m}$ and either

(a) $v \in \mathbf{Z}^{+}$and $f \in \mathbf{C}^{v-1,1}$, or

(b) $f \in \mathbf{C}^{v}$,

then $f(E)$ is m-null.

The crux of our problem is thus to eliminate from Norton's Lemma the assumption that $E$ is $n$-null; for this purpose, we need a further result of Norton:

The Generalized Morse Criticality Theorem (Norton). Let $n$ and $p$ be positive integers, $t \geq 1$ a real number, $E \subset \mathbf{R}^{n}$.

(a) There are subsets $E_{j}, j=0,1, \ldots$, of $E$ with $E=\bigcup E_{j}$ such that $E_{0}$ is countable and any $g \in \mathbf{C}^{p, 1}\left(\mathbf{R}^{n}, \mathbf{R}\right)$ critical on $E$ satisfies, for each $j$,

$$
|g(x)-g(y)| \leq M_{j}|x-y|^{p+1}
$$

for all $x, y \in E_{j}$ and some $M_{j}>1$.

(b) There are subsets $E_{j}^{\prime}, j=0,1, \ldots$ of $E$ with $E=\bigcup E_{j}^{\prime}$ such that $E_{0}$ is countable and any $g \in \mathbf{C}^{t}\left(\mathbf{R}^{n}, \mathbf{R}\right)$ critical on $E$ satisfies, for each $j$,

$$
|g(x)-g(y)| \leq M_{j}|x-y|^{t}
$$

for all $x, y \in E_{j}^{\prime}$ and some $M_{j}>1$. 
Finally, recall that an element $x \in \mathbf{R}^{n}$ is called a density point of $E \subset \mathbf{R}^{n}$ if for any sequence of cubes $\{Q\}$ decreasing to $x$, the limit

$$
\lim _{Q \searrow x} \frac{l_{n}(E \cap Q)}{l_{n}(Q)}=1
$$

holds. A familiar theorem from analysis asserts that almost all points of a measurable set are density points.

We now prove Lemma 1(a). Let $n, m$ be positive integers, define $v=n / m$, and suppose $E \subset \mathbf{R}^{n}$ is a rank 0 subset for a map $f: \mathbf{R}^{n} \rightarrow \mathbf{R}^{m}$. Our objective is to show that $f(E)$ is $m$-null provided $v$ is an integer and $f \in \mathbf{C}^{v-1,1}$.

Choose a decomposition $\left\{E_{j}\right\}$ of $E$ as in part (a) of the Generalized Morse Theorem. Clearly we may suppose that each $E_{j}$ is $n$-finite; we now demonstrate that $f\left(E_{j}\right)$ is $m$-null for all $j$ :

(a) Since $E_{0}$ is countable, $f\left(E_{0}\right)$ is $m$-null.

(b) Fix $j \geq 1$ and a positive integer $P$.

(i) By part (a) of Norton's Lemma, we may assume that every point of $E_{j}$ is a density point of $E_{j}$. Choose $x_{0} \in E_{j}$, and let $Q$ be a cube of edge $\lambda$ containing $x_{0}$ and small enough that any cube $Q^{\prime} \subset Q$ of edge $\lambda(2 n P)^{-1}$ intersects $E_{j}$. If $x, y$ are any two elements of $Q \cap E_{j}$, there evidently exists a covering of the line segment $\overline{x y}$ by at most $2 n P$ such subcubes of $Q$. Consequently, there exists a sequence $\left\{x_{i}\right\}_{i=0}^{2 n P} \subset E$ containing $x, y$ and satisfying $\left|x_{i}-x_{i+1}\right|<\lambda / P$ for $i=1, \ldots, 2 n P-1$. By part (a) of the Generalized Morse Theorem applied to each component function of $f$, there exists a constant $M>1$ depending only on $f$ and $E_{j}$ such that

$$
\begin{aligned}
|f(x)-f(y)| & \leq\left|f\left(x_{1}\right)-f\left(x_{2}\right)\right|+\cdots+\left|f\left(x_{2 n P-1}\right)-f\left(x_{2 n P}\right)\right| \\
& \leq M\left(\left|x_{1}-x_{2}\right|^{v}+\cdots+\left|x_{2 n P-1}-x_{2 n P}\right|^{v}\right)<2 n M \lambda^{v} P^{1-v} .
\end{aligned}
$$

Thus $l_{m}\left(f\left(Q \cap E_{j}\right)\right) \leq(2 n M)^{m} \lambda^{n} P^{m(1-v)}=(2 n M)^{m} P^{m(1-v)} l_{n}(Q)$.

(ii) Since for a given $P$ the family of all cubes $Q$ chosen as above for all $x_{0} \in E_{j}$ evidently comprises a Vitali family for $E_{j}$, there exists a sequence $\left\{Q_{l}\right\}$ of such cubes satisfying $l_{n}\left(E_{j} \backslash \cup Q_{l}\right)=0$ and $\sum l_{n}\left(Q_{l}\right)<2 l_{n}\left(E_{j}\right)$.

Applying part (a) of Norton's Lemma once again, it follows that

$$
\begin{aligned}
l_{m}\left(f\left(E_{j}\right)\right) & \leq \sum l_{m}\left(f\left(Q_{l} \cap E_{j}\right)\right) \leq(2 n M)^{m} P^{m(1-v)} \sum l_{n}\left(Q_{l}\right) \\
& <2(2 n M)^{m} P^{m(1-v)} l_{n}\left(E_{j}\right) .
\end{aligned}
$$

Since $P$ may be chosen arbitrarily large, $f\left(E_{j}\right)$ is $m$-null, and the assertion follows.

To prove Lemma 1(b), choose a decomposition $\left\{E_{j}^{\prime}\right\}$ of $E$ as in part (b) of the Generalized Morse Theorem, and proceed analogously.

\section{ACKNOWLEDGMENTS}

I am happy to thank R. Kaufman for his suggestions in [6] and C. Pugh for helpful criticisms of the manuscript. 


\section{REFERENCES}

1. S. M. Bates, On the image size of singular maps. I, Proc. Amer. Math. Soc. 114 (1992), 699-705.

2. __ On the image size of singular maps. II, preprint.

3. H. Federer, Geometric measure theory, Grundlehren Math. Wiss., vol. 153, Springer-Verlag, New York, 1969.

4. E. L. Grinberg, On the smoothness hypothesis in Sard's Theorem, Amer. Math. Monthly 47 (1985), 299-304.

5. R. Kaufman, A singular map of a cube onto a square, J. Differential Geom. 14 (1979), 593-594.

6. , private communication, August 1990.

7. A. Norton, A critical set with nonnull image has large Hausdorff dimension, Trans. Amer. Math. Soc. 296 (1986), 367-376.

8. The fractal geometry of critical sets with nonnull image and the differentiability of functions, Ph.D. Thesis, Univ. of California, Berkeley, 1987.

9. A. Sard, The measure of the critical values of differentiable maps, Bull. Amer. Math. Soc. $\mathbf{4 8}$ (1942), 883-890.

10. S. Sternberg, Lectures on differential geometry, Prentice-Hall, Englewood Cliffs, NJ, 1964.

11. H. Whitney, A function not constant on a connected set of critical points, Duke Math J. 1 (1935), 514-517.

12. Y. Yomdin, The geometry of critical and near-critical values of differentiable mappings, Math. Ann. 264 (1983), 495-515.

Department of Mathematics, University of California at Berkeley, Berkeley, CaliFORNIA 94720 